Kragujevac Journal of Mathematics

Volume 44(4) (2020), Pages 495-508.

\title{
RESULTS ON TAUBERIAN THEOREM FOR CESÀRO SUMMABLE DOUBLE SEQUENCES OF FUZZY NUMBERS
}

\author{
B. B. JENA ${ }^{1}$, S. K. PAIKRAY ${ }^{1}$, P. PARIDA ${ }^{2}$, AND H. DUTTA ${ }^{3}$
}

\begin{abstract}
The paper aims to establish new results on Tauberian theorem for Cesàro summability of double sequences of fuzzy numbers, and thus to extend and unify several results in the available literature. Further, a number of special cases, corollaries and illustrative example in support of the investigation of this paper are also presented.
\end{abstract}

\section{Introduction AND PRELIMINARIES}

The notion of the fuzzy set was introduced by Zadeh [19]. Matloka [10] has established bounded and convergent sequences of fuzzy numbers and proved that every convergent sequence is bounded. Nanda [12] has studied the spaces of bounded and convergent sequences of fuzzy numbers and proved that every Cauchy sequence of fuzzy numbers is convergent. Subrahmanyam [13] has presented Cesàro summability of sequences of fuzzy numbers and established Tauberian hypotheses identified with the Cesàro summability method. Talo and Çanak [15] introduced necessary and sufficient Tauberian conditions, under which convergence follows from Cesàro convergence of sequences of fuzzy numbers. Altin et al. [1] studied the concept of statistical summability by $(C, 1)$-mean for sequences of fuzzy numbers and obtained a Tauberian theorem on that basis. Talo and Başar [14] introduced the concept of slow decreasing sequence for fuzzy numbers and have shown that Cesàro summable sequence $\left(X_{n}\right)$ is convergent, if $\left(X_{n}\right)$ is slowly decreasing. Recently, Çanak [2] has established the concept of the slow oscillation (that is, both slowly decreasing and slowly increasing)

Key words and phrases. Double Cesàro summability, slow oscillation, Tauberian condition, sequence of fuzzy numbers.

2010 Mathematics Subject Classification. Primary: 40G05, 40E05. Secondary: 40A10, 03E72.

DOI 10.46793/KgJMat2004.495J

Received: March 15, 2018.

Accepted: June 11, 2018. 
sequences for fuzzy numbers and have shown that Cesàro summable sequence $\left(X_{n}\right)$ is convergent if $\left(X_{n}\right)$ is slowly oscillating.

Let $D$ denote the set of all closed and bounded intervals $X=\left[x_{1}, x_{2}\right]$ on the real line $\mathbb{R}$. For $X, Y \in D$, we define

$$
d(X, Y)=\max \left\{\left|x_{1}-y_{1}\right|,\left|x_{2}-y_{2}\right|\right\},
$$

where

$$
X=\left[x_{1}, x_{2}\right], \quad Y=\left[y_{1}, y_{2}\right]
$$

It is surely understood that $(D, d)$ is a complete metric space.

A fuzzy number $X$ is a fuzzy set on $\mathbb{R}$ and is a mapping $X: \mathbb{R} \rightarrow[0,1]$ associating each number $t$ with its grade of membership $X(t)$.

A fuzzy number $X$ is said to be convex, if

$$
X(t)=\min \{X(s), X(r)\}, \quad s<t<r .
$$

If there exists $t_{0} \in \mathbb{R}$, such that $X\left(t_{0}\right)=1$, then the fuzzy number $X$ is called normal. A fuzzy number $X$ is said to be upper semi-continuous if, for each $\epsilon>0$, we have

$$
X^{-1}([0, x+\epsilon))
$$

for all $x \in[0,1])$, is open in the usual topology of $\mathbb{R}$. The set of all upper semicontinuous, normal, convex fuzzy number is denoted by $\mathbb{R}([0,1])$. For $\alpha \in(0,1]$, the $\alpha$ - level set of fuzzy number $X$ denoted by $X^{\alpha}$ is defined by

$$
X^{\alpha}=\{t \in \mathbb{R}: X(t) \geqq \alpha\} .
$$

The set $X^{0}$ is defined as the closure of the following set

$$
\{t \in \mathbb{R}: X(t)>0\} .
$$

We define,

by

$$
\bar{d}: \mathbb{R}([0,1]) \times \mathbb{R}([0,1]) \rightarrow \mathbb{R}_{+} \cup\{0\},
$$

$$
\bar{d}(X, Y)=\sup _{0 \leqq \alpha \leqq 1} d\left(X^{\alpha}, Y^{\alpha}\right) .
$$

\section{Definitions And Motivation}

A double sequence $\left(X_{m n}\right)$ of fuzzy numbers is a function, $X: \mathbb{N} \cup\{0\} \times \mathbb{N} \cup\{0\} \rightarrow$ $\mathbb{R}([0,1])$ and is said to be convergent to a fuzzy number $X_{0}$ if, for every $\epsilon>0$, there exist a positive integer $n_{0}$ such that

$$
\bar{d}\left(X_{m n}, X_{0}\right)<\epsilon, \quad \text { for all } m, n \geqq n_{0} .
$$

We define,

and

$$
\begin{aligned}
& \Delta_{n} X_{m n}=\bar{d}\left(X_{m n}, X_{m, n-1}\right) \\
& \Delta_{m} X_{m n}=\bar{d}\left(X_{m n}, X_{m-1, n}\right)
\end{aligned}
$$

$$
\Delta_{m, n} X_{m n}=\bar{d}\left(X_{m n}, X_{m-1, n}\right)-\bar{d}\left(X_{m, n-1}, X_{m-1, n-1}\right), \quad X_{-1}=0
$$


A double sequence $\left(X_{m n}\right)$ of fuzzy numbers is said to be bounded, if there exists a positive number $\mathcal{K}>0$ such that

$$
\bar{d}\left(X_{m n}, X_{0}\right) \leqq \mathcal{K}, \quad \text { for all } m, n \in \mathbb{N} \cup\{0\} .
$$

The Cesàro means $(C, 1)$ of sequence $\left(X_{n}\right)$ of fuzzy numbers are defined by

$$
\sigma_{n}=\frac{1}{n+1} \sum_{j=0}^{n} X_{j}, \quad \text { for all } n \in \mathbb{N} \cup\{0\} .
$$

A sequence $\left(X_{n}\right)$ of fuzzy numbers is Cesàro summable to a fuzzy number $L$ if, for every $\epsilon>0$, we have (see [2])

$$
\bar{d}\left(\sigma_{n}, L\right)<\epsilon, \quad n \rightarrow \infty .
$$

Similarly, the Cesàro means $(C, 1,1)$ of double sequences $\left(X_{m n}\right)$ of fuzzy numbers are defined by

$$
\sigma_{m n}^{(1,1)}(X)=\frac{1}{(m+1)(n+1)} \sum_{p=0}^{m} \sum_{q=0}^{n} X_{p q}=\sum_{p=1}^{m} \sum_{q=1}^{n} \frac{Y_{p q}^{(1,1)}}{p q}+X_{00}
$$

(see [11]). Analogous to equation $(2.1)$, we may define the $(C, 1,0)$ and $(C, 0,1)$-means of sequences $\left(X_{m n}\right)$ are

$$
\sigma_{m n}^{(1,0)}(X)=\frac{1}{m+1} \sum_{p=0}^{m} X_{p n} \quad \text { and } \quad \sigma_{m n}^{(0,1)}(X)=\frac{1}{n+1} \sum_{q=0}^{n} X_{m q}
$$

respectively.

Then we say that, a double sequence $X=\left(X_{m n}\right)$ of fuzzy numbers is $(C, 1,1)$ summable to a fuzzy number $L$ if, for every $\epsilon>0$, we have

$$
\bar{d}\left(\sigma_{m n}^{(1,1)}(X), L\right)<\epsilon, \quad \text { for all } m, n \rightarrow \infty .
$$

Similarly, we say that it is $(C, 1,0)$-summable to a fuzzy number $L$ if, for every $\epsilon>0$, we have

$$
\bar{d}\left(\sigma_{m n}^{(1,0)}(X), L\right)<\epsilon, \quad \text { for all } m, n \rightarrow \infty
$$

and $(C, 0,1)$-summable to a fuzzy number $L$ if, for every $\epsilon>0$, we have

$$
\bar{d}\left(\sigma_{m n}^{(1,0)}(X), L\right)<\epsilon, \quad \text { for all } m, n \rightarrow \infty .
$$

Now, for each non-negative integers $k$ and $r$, we may define $\sigma_{m n}^{(k r)}(X)$ as follows:

$$
\sigma_{m n}^{(k r)}(X)= \begin{cases}\frac{1}{(m+1)(n+1)} \sum_{p=0}^{m} \sum_{q=0}^{n} \sigma_{p q}^{(k-1, r-1)}, & k, r \geq 1, \\ X_{m n}, & k, r=0 .\end{cases}
$$

A double sequence $X=\left(X_{m n}\right)$ of fuzzy numbers is said to be $(C, k, r)$-summable to a fuzzy number $L$ if, for every $\epsilon>0$, we have

$$
\bar{d}\left(\sigma_{m n}^{(k r)}(X), L\right)<\epsilon, \quad \text { for all } m, n \rightarrow \infty
$$


Remark 2.1. If $k=1$ and $r=1$, then $(C, k, r)$-summability reduces to $(C, 1,1)$ summability. Furthermore, if $k \neq 0$ and $r=0$ then $(C, k, r)$-summability reduces to $(C, k, 0)$-summability. Finally, if $k=0$ and $r \neq 0$ then $(C, k, r)$-summability reduces to $(C, 0, r)$-summability.

Note that here, Cesàro summability of $X=\left(X_{m n}\right)$ refers $(C, 1,1)$ and $(C, k, r)$ summability of $X=\left(X_{m n}\right)$.

It may also be noted that, the convergence of a double sequence $X=\left(X_{m n}\right)$ of fuzzy numbers implies the Cesàro summability of $X=\left(X_{m n}\right)$, but the converse is not generally true.

For example, consider a function $f(x, y)=e^{2 x} \sin (3 y)$; the sequence $\left(X_{m n}\right)$ of fuzzy numbers which is the sequence of coefficients in the Taylor's series expansion of the function $f(x, y)$ about origin is Cesàro summable but not convergent.

For the proof of converse part, certain conditions are presented in terms of oscillatory behavior of double sequence $X=\left(X_{m n}\right)$ of fuzzy numbers.

Let us define $\left(X_{m n}\right)$ as

$$
X_{m n}=Y_{m n}^{(1,1)}+\sum_{p=1}^{m} \sum_{q=1}^{n} \frac{Y_{p q}^{(1,1)}}{p q}+X_{00}, \quad m, n \in \mathbb{N},
$$

where

$$
X_{m n}-\sigma_{m n}^{(1,1)}(X)=Y_{m n}^{(1,1)}(\Delta X)=\frac{1}{(m+1)(n+1)} \sum_{p=0}^{m} \sum_{q=0}^{n} p q\left(\Delta_{p, q} X_{p q}\right)
$$

(see [9]). Moreover, in analogy to Kronecker identity for a single sequence of fuzzy numbers, we can write

$$
Y_{m n}^{(1,0)}(\Delta X)=\frac{1}{(m+1)} \sum_{p=0}^{m} p\left(\Delta_{p}, X_{p, n}\right)
$$

and

$$
Y_{m n}^{(0,1)}(\Delta X)=\frac{1}{(n+1)} \sum_{q=0}^{n} q\left(\Delta_{q}, X_{m, q}\right)
$$

as the $(C, 1,0)$-mean of the sequence $\left(m \Delta_{m} X_{m n}\right)$ of fuzzy numbers and the $(C, 0,1)$ mean of the sequence $\left(n \Delta_{n} X_{m n}\right)$ fuzzy number respectively.

Furthermore, as the sequence $Y_{m n}^{(1,1)}\left(\Delta_{m n} X_{m n}\right)$ of fuzzy numbers is the $(C, 1,1)$ mean of the sequence $m n\left(\Delta_{m n} X_{m n}\right)$ of fuzzy number, the sequence $m n\left(\Delta_{m n} X_{m n}\right)$ is $(C, 1,1)$-summable to a fuzzy number $L$, whenever

$$
\bar{d}\left(Y_{m n}^{(1,1)}\left(\Delta_{m n} X_{m n}\right), L\right)<\epsilon, \quad \text { for all } m, n \rightarrow \infty .
$$

For each non-negative integers $k$ and $r$, let us define $Y_{m n}^{(k, r)}(\Delta X)$ as follows:

$$
Y_{m n}^{(k, r)}(\Delta X)= \begin{cases}\frac{1}{(m+1)(n+1)} \sum_{p=0}^{m} \sum_{q=0}^{n} Y_{p q}^{(k-1, r-1)}, & k, r \geq 1, \\ m n\left(\Delta_{m, n} X_{m n}\right), & k, r=0 .\end{cases}
$$


The sequence $m n\left(\Delta_{m n} X_{m n}\right)$ of fuzzy numbers is said to be $(C, k, r)$-summable to a fuzzy numbers $L$ if, for every $\epsilon>0$, we have

$$
\bar{d}\left(Y_{m n}^{(k r)}\left(\Delta_{m n} X_{m n}\right), L\right)<\epsilon, \text { for all } m, n \rightarrow \infty .
$$

Remark 2.2. If $k=1$ and $r=1$, then $(C, k, r)$-summabllity reduces to $(C, 1,1)$ summability. Furthermore, if $k \neq 0$ and $r=0$, then $(C, k, r)$-summabllity reduces to $(C, k, 0)$-summability. Finally, if $k=0$ and $r \neq 0$, then $(C, k, r)$-summabllity reduces to $(C, 0, r)$-summability.

Next, we present the De la Vallée Poussin mean of double sequence $\left(X_{m n}\right)$ of fuzzy numbers for sufficiently large nonnegative integers $m, n$ for $\lambda>1$ and $0<\lambda<1$

$$
\tau_{m n}(X)=\frac{1}{([\lambda m]-m)([\lambda n]-n)} \sum_{i=m+1}^{[\lambda m]} \sum_{j=n+1}^{[\lambda n]} X_{i j}
$$

and

respectively.

$$
\tau_{m n}(X)=\frac{1}{(m-[\lambda m])(n-[\lambda n])} \sum_{i=\lambda m+1}^{m} \sum_{j=\lambda n+1}^{n} X_{i j}
$$

A single sequence $X=\left(X_{n}\right)$ of fuzzy numbers is slowly oscillating (in the sense of Stanojević) if, (see [18])

$$
\lim _{\lambda \rightarrow 1^{+}} \limsup _{n} \max _{n+1 \leqq k \leqq[\lambda n]} \bar{d}\left(X_{k}, X_{n}\right)=0 .
$$

Similarly, we may write a double sequence $X=\left(X_{m n}\right)$ of fuzzy numbers is slowly oscillating (in the sense of Stanojević) if,

$$
\lim _{\lambda \rightarrow 1^{+}} \limsup _{m, n} \max _{m+1, n+1 \leqq i, j \leqq[\lambda m],[\lambda n]} \bar{d}\left(\sum_{u=m+1}^{i} \sum_{v=n+1}^{j} \Delta_{u, v} X_{u v}, 0\right) \leqq \epsilon .
$$

Recently, few researchers have investigated on sequences and sequences of fuzzy numbers for proving Tauberian theorems. Different classes of sequences and sequences of fuzzy numbers have been introduced and studied by Tripathy et al. [17], Dutta [3], Dutta [4], Dutta and Bilgin [6], Tripathy and Debnath [16], Dutta and Başar [5], Jena et al. [7], Jena et al. [8] and many others. Recently, Çanak [2] has introduced Tauberian theorem for Cesàro summability of sequences of fuzzy numbers.

Motivated essentially by the above-mentioned works, here we wish to present the (presumably new) the notion of $(C, 1,1)$-summability of a double sequences of fuzzy numbers defined in (2.3).

\section{Tauberian Theorems for Cesàro Mean}

Theorem 3.1. If the double sequence $\left(X_{m n}\right)$ of fuzzy number is $(C, 1,1)$-summable to a fuzzy number $L$ and $\left(X_{m n}\right)$ is slowly oscillating (in the sense of Stanojević), then

$$
\bar{d}\left(X_{m n}, L\right)<\epsilon, \quad \text { for all } m, n \rightarrow \infty .
$$


To prove the above theorem, we need the help of the following lemmas.

Lemma 3.1. A double sequence $X=\left(X_{m n}\right)$ of fuzzy numbers is slowly oscillating if and only if $\left(Y_{m n}^{(1,1)}\right)$ is slowly oscillating and bounded.

Proof. Let $X=\left(X_{m n}\right)$ is slowly oscillating. Initially, let us show that

$$
\bar{d}\left(V_{m n}^{(1,1)}, 0\right)=O(1) .
$$

We have by definition of slow oscillation, for $\lambda>1$

$$
\lim _{\lambda \rightarrow 1^{+}} \limsup _{m, n} \max _{m+1, n+1 \leqq i, j \leqq[\lambda m, \lambda n]} \bar{d}\left(\sum_{u=m+1}^{i} \sum_{v=n+1}^{j} \Delta_{u, v} X_{u v}, 0\right) \leqq \epsilon
$$

and let us rewrite the finite sum

$$
\sum_{i=1}^{m} \sum_{j=1}^{n} i j \Delta X_{i j}
$$

as the series

$$
\sum_{u=0}^{\infty} \sum_{v=0}^{\infty} \sum_{\frac{m}{2^{u+1}}, \frac{n}{2^{v+1}} \leqq i, j \leqq \frac{m}{2^{u}}, \frac{n}{2^{v}}} i j \Delta X_{i j}
$$

Clearly,

$$
\begin{aligned}
\bar{d}\left(\sum_{i=1}^{m} \sum_{j=1}^{n} i j \Delta X_{i j}, 0\right) & \leqq \bar{d}\left(\sum_{u=0}^{\infty} \sum_{v=0}^{\infty} \sum_{\frac{m}{2^{u+1}}, \frac{n}{2^{v+1}} \leqq i, j<\frac{m}{2^{u}}, \frac{n}{2^{v}}} i j \Delta X_{i j}, 0\right) \\
& \leqq \bar{d}\left(\sum_{u=0}^{\infty} \sum_{v=0}^{\infty} \frac{m n}{2^{u+v}}, 0\right)
\end{aligned}
$$

and

$$
\begin{aligned}
& \max _{\frac{m}{2^{u+1}}+1, \frac{n}{2^{v+1}}+1 \leqq i, j \leqq}^{\left.\frac{\lambda m}{2^{i+1}}, \frac{\lambda n}{2^{j+1}}\right]} \bar{d}\left(\sum_{u=\frac{m}{2^{u+1}}+1}^{i} \sum_{v=\frac{n}{2^{v+1}}+1}^{j} \Delta_{u, v} X_{u v}, 0\right) \\
& \leq m n C\left(\sum_{u=0}^{\infty} \sum_{v=0}^{\infty} \frac{1}{2^{u+v}}\right)=m n C^{*}, \quad C^{*}>0 .
\end{aligned}
$$

Consequently, we have

$\bar{d}\left(Y_{m n}^{(1,1)}(\Delta X), 0\right)=\bar{d}\left(\frac{1}{(m+1)(n+1)} \sum_{p=0}^{m} \sum_{q=0}^{n} p q\left(\Delta_{p, q} X_{p q}\right), 0\right)=O(1), \quad m, n \rightarrow \infty$.

Since,

$$
\left\{\sigma_{m n}^{(1,1)}(X)=\sum_{p=1}^{m} \sum_{q=1}^{n} \frac{Y_{p q}^{(1,1)}}{p q}+X_{00}\right\}
$$

is slowly oscillating; so $\left(Y_{m n}^{(1,1)}\right)$ is oscillating slowly. 
To prove the converse part, consider $\left(Y_{m n}^{(1,1)}\right)$ is bounded and slowly oscillating. Now the boundedness of $\left(Y_{m n}^{(1,1)}\right)$ implies that $\sigma_{m n}^{(1,1)}(X)$ is slowly oscillating. Furthermore, $\left(Y_{m n}^{(1,1)}\right)$ being oscillating slowly, so by Kronecker identity $(2.4),\left(X_{m n}\right)$ is oscillating slowly. Which completes the proof of Lemma 3.1.

Next, we represent $\bar{d}\left(X_{m n}, \sigma_{m n}^{(11)}(X)\right)$ under two different cases in the following lemma.

Lemma 3.2. Let $X=\left(X_{m n}\right)$ be a sequence of fuzzy numbers with $m, n$ sufficiently large, then we have the following.

(i) For $\lambda>1$

$$
\begin{aligned}
\bar{d}\left(X_{m n}, \sigma_{m n}^{(1,1)}(X)\right)= & \frac{([\lambda m]+1)([\lambda n]+1)}{([\lambda m]-m)([\lambda n]-n)}\left\{\bar{d}\left(\sigma_{[\lambda m],[\lambda n]}^{(1,1)}(X), \sigma_{[\lambda m], n}^{(1,1)}(X)\right)\right. \\
& \left.-\bar{d}\left(\sigma_{m,[\lambda n]}^{(1,1)}(X), \sigma_{m n}^{(1,1)}(X)\right)\right\} \\
& +\frac{[\lambda m]+1}{[\lambda n]-m} \bar{d}\left(\sigma_{[\lambda m], n}^{(1,1)}(X), \sigma_{m, n}^{(1,1)}(X)\right) \\
& +\frac{[\lambda n]+1}{[\lambda m]-m} \bar{d}\left(\sigma_{m,[\lambda n]}^{(1,1)}(X), \sigma_{m n}^{(1,1)}(X)\right) \\
& -\frac{1}{([\lambda m]-m)([\lambda n]-n)} \bar{d}\left(\sum_{i=m+1}^{[\lambda m]} \sum_{j=n+1}^{[\lambda n]}\left(X_{i j}, X_{m n}\right)\right)
\end{aligned}
$$

(ii) For $0<\lambda<1$

$$
\begin{aligned}
\bar{d}\left(X_{m n}, \sigma_{m n}^{(1,1)}(X)\right)= & \frac{([\lambda m]+1)([\lambda n]+1)}{(m-[\lambda m])(n-[\lambda n])}\left\{\bar{d}\left(\sigma_{m n}^{(1,1)}(X), \sigma_{[\lambda m], n}^{(1,1)}(X)\right)\right. \\
& \left.-\bar{d}\left(\sigma_{m,[\lambda n]}^{(1,1)}(X), \sigma_{[\lambda m],[\lambda n]}^{(1,1)}(X)\right)\right\} \\
& +\frac{[\lambda m]+1}{m-[\lambda m]} \bar{d}\left(\sigma_{m, n}^{(1,1)}(X), \sigma_{[\lambda m], n}^{(1,1)}(X)\right) \\
& +\frac{[\lambda n]+1}{n-[\lambda n]} \bar{d}\left(\sigma_{m n}^{(1,1)}(X), \sigma_{m,[\lambda n]}^{(1,1)}(X)\right) \\
& -\frac{1}{(m-[\lambda m])(n-[\lambda n])} \bar{d}\left(\sum_{i=[\lambda m]+1}^{m} \sum_{j=[\lambda n]+1}^{n}\left(X_{m n}, X_{i j}\right)\right) .
\end{aligned}
$$

Proof. We have by De la Vallée Poussin mean of double sequence $\left(X_{m n}\right)$ of fuzzy numbers

$$
\tau_{m n}(X)=\frac{1}{([\lambda m]-m)([\lambda n]-n)} \sum_{i=m+1}^{[\lambda m]} \sum_{j=n+1}^{[\lambda n]} X_{i j}
$$




$$
\begin{aligned}
& =\frac{1}{([\lambda m]-m)([\lambda n]-n)}\left\{\bar{d}\left(\sum_{i=0}^{[\lambda m]}, \sum_{i=0}^{[m]}\right) \bar{d}\left(\sum_{j=0}^{[\lambda n]}, \sum_{j=0}^{[n]}\right)\right\} X_{i j} \\
& =\frac{1}{([\lambda m]-m)([\lambda n]-n)}\left\{\bar{d}\left(\sum_{i=0}^{[\lambda m]} \sum_{j=0}^{[\lambda n]}, \sum_{i=0}^{[\lambda m]} \sum_{j=0}^{[n]}\right) X_{i j}-\bar{d}\left(\sum_{i=0}^{[m]} \sum_{j=0}^{[\lambda n]}, \sum_{i=0}^{[m]} \sum_{j=0}^{[n]}\right) X_{i j}\right\} \\
& =\frac{1}{([\lambda m]-m)([\lambda n]-n)}\left\{([\lambda n]+1)([\lambda m]+1) \sigma_{[\lambda m],[\lambda n]}^{(1,1)}\right. \\
& \left.-([\lambda m]+1)(n+1) \sigma_{[\lambda m], n}^{(1,1)}\right\}-\frac{1}{([\lambda m]-m)([\lambda n]-n)} \\
& -\left\{(m+1)([\lambda n]+1) \sigma_{m,[\lambda n]}^{(1,1)}-(m+1)(n+1) \sigma_{m n}^{1,1}\right\} \\
& =\frac{([\lambda m]+1)([\lambda n]+1)}{([\lambda m]-m)([\lambda n]-n)} \sigma_{[\lambda m],[\lambda n]}^{(1,1)}-\left\{\frac{([\lambda m]+1)([\lambda n]+1)}{([\lambda m]-m)([\lambda n]-n)} \sigma_{[\lambda m], n}^{(1,1)}\right. \\
& \left.-\frac{([\lambda m]+1)}{([\lambda m]-m)} \sigma_{[\lambda m], n}^{(1,1)}\right\} \\
& -\left\{\frac{([\lambda m]+1)([\lambda n]+1)}{([\lambda m]-m)([\lambda n]-n)} \sigma_{m,[\lambda n]}^{(1,1)}-\frac{([\lambda n]+1)}{([\lambda n]-n)} \sigma_{m,[\lambda n]}^{(1,1)}\right\} \\
& +\left\{\frac{([\lambda m]+1)([\lambda n]+1)}{([\lambda m]-m)([\lambda n]-n)} \sigma_{m, n}^{(1,1)}-\frac{([\lambda m]+1)}{([\lambda m]-m)} \sigma_{m, n}^{(1,1)}\right. \\
& \left.-\frac{([\lambda n]+1)}{([\lambda n]-n)} \sigma_{m, n}^{(1,1)}+\sigma_{m, n}^{(1,1)}\right\} \text {. }
\end{aligned}
$$

Which implies

$$
\begin{aligned}
\tau_{m n}-\sigma_{m, n}^{(1,1)}= & \frac{([\lambda m]+1)([\lambda n]+1)}{([\lambda m]-m)([\lambda n]-n)}\left\{\bar{d}\left(\sigma_{[\lambda m],[\lambda n]}^{(1,1)}, \sigma_{[\lambda m], n}^{(1,1)}\right)-\bar{d}\left(\sigma_{m,[\lambda n]}^{(1,1)}, \sigma_{m, n}^{(1,1)}\right)\right\} \\
& +\frac{([\lambda m]+1)}{([\lambda m]-m)} \bar{d}\left(\sigma_{[\lambda m], n}^{(1,1)}, \sigma_{m, n}^{(1,1)}\right)+\frac{([\lambda n]+1)}{([\lambda n]-n)} \bar{d}\left(\sigma_{m,[\lambda n]}^{(1,1)}, \sigma_{m, n}^{(1,1)}\right) .
\end{aligned}
$$

Also,

$$
X_{m n}=\tau_{m n}-\frac{1}{([\lambda m]-m)([\lambda n]-n)} \bar{d}\left(\sum_{i=m+1}^{[\lambda m]} \sum_{j=n+1}^{[\lambda n]}\left(X_{i j}, X_{m n}\right)\right) .
$$

Subtracting $\left(\sigma_{[\lambda m],[\lambda n]}^{(1,1)}\right)$ from the above identity, we have

$$
\begin{aligned}
& \bar{d}\left(X_{m n}, \sigma_{m n}^{(1,1)}(X)\right) \\
= & \bar{d}\left(\tau_{m n}(X), \sigma_{[\lambda m],[\lambda n]}^{(1,1)}\right)-\frac{1}{([\lambda m]-m)([\lambda n]-n)} \bar{d}\left(\sum_{i=m+1}^{[\lambda m]} \sum_{j=n+1}^{[\lambda n]}\left(X_{i j}, X_{m n}\right)\right)
\end{aligned}
$$




$$
\begin{aligned}
= & \frac{([\lambda m]+1)([\lambda n]+1)}{([\lambda m]-m)([\lambda n]-n)}\left\{\bar{d}\left(\sigma_{[\lambda m],[\lambda n]}^{(1,1)}(X), \sigma_{[\lambda m], n}^{(1,1)}(X)\right)-\bar{d}\left(\sigma_{m,[\lambda n]}^{(1,1)}(X), \sigma_{m n}^{(1,1)}(X)\right)\right\} \\
& +\frac{[\lambda m]+1}{[\lambda n]-m} \bar{d}\left(\sigma_{[\lambda m], n}^{(1,1)}(X), \sigma_{m, n}^{(1,1)}(X)\right)+\frac{[\lambda n]+1}{[\lambda m]-m} \bar{d}\left(\sigma_{m,[\lambda n]}^{(1,1)}(X), \sigma_{m n}^{(1,1)}(X)\right) \\
& -\frac{1}{([\lambda m]-m)([\lambda n]-n)} \bar{d}\left(\sum_{i=m+1}^{[\lambda m]} \sum_{j=n+1}^{[\lambda n]}\left(X_{i j}, X_{m n}\right)\right) .
\end{aligned}
$$

Which establish (i). Next, the proof of (ii) is similar to (i).

\section{Proof of Theorem 1.}

Proof. Let $\left(X_{m n}\right)$ is $(C, 1,1)$-summable to a fuzzy number $L$, this implies $\sigma_{m n}^{(1,1)}$ is $(C, 1,1)$-summable to a fuzzy number $L$. Now from equation $(2.4)$, we have $\left(Y_{m n}^{(1,1)}\right)$ is $(C, 1,1)$-summable to zero. Thus by Lemma 3.1, $\left(Y_{m n}^{(1,1)}\right)$ oscillating slowly. Again by Lemma 3.2 (i), we get

$$
\begin{aligned}
\bar{d}\left(Y_{m n}^{(1,1)}, \sigma_{m n}^{(1,1)}\left(Y_{m n}^{(1,1)}\right)\right)= & \frac{([\lambda m]+1)([\lambda n]+1)}{([\lambda m]-m)([\lambda n]-n)}\left\{\bar{d}\left(\sigma_{[\lambda m],[\lambda n]}^{(1,1)}\left(Y_{m n}^{(1,1)}\right), \sigma_{[\lambda m], n}^{(1,1)}\left(Y_{m n}^{(1,1)}\right)\right)\right. \\
& \left.-\bar{d}\left(\sigma_{m,[\lambda n]}^{(1,1)}\left(Y_{m n}^{(1,1)}\right), \sigma_{m n}^{(1,1)}\left(Y_{m n}^{(1,1)}\right)\right)\right\} \\
& +\frac{[\lambda m]+1}{[\lambda n]-m} \bar{d}\left(\sigma_{[\lambda m], n}^{(1,1)}\left(Y_{m n}^{(1,1)}\right), \sigma_{m, n}^{(1,1)}\left(Y_{m n}^{(1,1)}\right)\right) \\
& +\frac{[\lambda n]+1}{[\lambda m]-m} \bar{d}\left(\sigma_{m,[\lambda n]}^{(1,1)}\left(Y_{m n}^{(1,1)}\right), \sigma_{m n}^{(1,1)}\left(Y_{m n}^{(1,1)}\right)\right) \\
& -\frac{1}{([\lambda m]-m)([\lambda n]-n)} \bar{d}\left(\sum_{i=m+1}^{[\lambda m]} \sum_{j=n+1}^{[\lambda n]}\left(Y_{i j}^{(1,1)}, Y_{m n}^{(1,1)}\right)\right) .
\end{aligned}
$$

It is easy to verify that for $\lambda>1$ and sufficiently large $n$

$$
\frac{([\lambda m]+1)([\lambda n]+1)}{([\lambda m]-m)([\lambda n]-n)}<\frac{([\lambda m]+1)([\lambda n]+1)}{([\lambda m]-1-m)([\lambda n]-1-n)}<\frac{4 \lambda^{2}}{(\lambda-1)^{2}} .
$$

Next, by (3.3)

$$
\begin{aligned}
\bar{d}\left(Y_{m n}^{(1,1)}, \sigma_{m n}^{(1,1)}\left(Y_{m n}^{(1,1)}\right)\right) \leqq & \frac{4 \lambda^{2}}{(\lambda-1)^{2}} \bar{d}\left(\tau_{m n}\left(Y_{m n}^{(1,1)}\right), \sigma_{[\lambda m],[\lambda n]}^{(1,1)}\left(Y_{m n}^{(1,1)}\right)\right) \\
& -\max _{m+1, n+1 \leqq i, j \leqq[\lambda m],[\lambda n]} \bar{d}\left(\sum_{j=n+1}^{[\lambda n]}\left(Y_{i j}^{(1,1)}, Y_{m n}^{(1,1)}\right)\right) .
\end{aligned}
$$


Taking limsup both sides of (3.4), we have

$\limsup _{m, n} \bar{d}\left(Y_{m n}^{(1,1)}, \sigma_{m n}^{(1,1)}\left(Y_{m n}^{(1,1)}\right)\right) \leqq \frac{4 \lambda^{2}}{(\lambda-1)^{2}} \limsup _{m, n} \bar{d}\left(\tau_{m n}\left(Y_{m n}^{(1,1)}\right), \sigma_{[\lambda m],[\lambda n]}^{(1,1)}\left(Y_{m n}^{(1,1)}\right)\right)$

$$
-\limsup _{m, n} \max _{m+1, n+1 \leqq i, j \leq[\lambda m],[\lambda n]} \bar{d}\left(\sum_{j=n+1}^{[\lambda n]}\left(Y_{i j}^{(1,1)}, Y_{m n}^{(1,1)}\right)\right) .
$$

Furthermore,

$$
\sigma_{[\lambda m],[\lambda n]}^{(1,1)}\left(Y_{m n}^{(1,1)}\right) \rightarrow 0, \quad m, n \rightarrow \infty,
$$

so first term in the right hand side of equation (3.5), must vanish.

This implies,

$$
\begin{aligned}
& \limsup _{m, n} \bar{d}\left(Y_{m n}^{(1,1)}, \sigma_{m n}^{(1,1)}\left(Y_{m n}^{(1,1)}\right)\right) \\
\leqq & \limsup _{m, n} \max _{m+1, n+1 \leqq i, j \leqq[\lambda m],[\lambda n]} \bar{d}\left(\sum_{j=n+1}^{[\lambda n]}\left(Y_{i j}^{(1,1)}, Y_{m n}^{(1,1)}\right)\right) .
\end{aligned}
$$

As $\lambda \rightarrow 1^{+}$in $(3.6)$, so we get

$$
\limsup _{m, n} \bar{d}\left(Y_{m n}^{(1,1)}, \sigma_{m n}^{(1,1)}\left(Y_{m n}^{(1,1)}\right)\right) \leqq 0 .
$$

It implies that,

$$
\bar{d}\left(Y_{m n}^{(1,1)}, 0\right)<\epsilon, \quad m, n \rightarrow \infty .
$$

Since $\left(X_{m n}\right)$ is summable to a fuzzy number $L$ by $(C, 1,1)$ mean and

$$
\bar{d}\left(Y_{m n}^{(1,1)}, 0\right)<\epsilon, \quad m, n \rightarrow \infty
$$

SO

$$
\bar{d}\left(X_{m n}, L\right)<\epsilon, \quad m, n \rightarrow \infty .
$$

Which completes the proof of the Theorem 3.1.

Corollary 3.1. If $\left(X_{m n}\right)$ is $(C, k, r)$-summable to a fuzzy number $L$ and $\left(X_{m n}\right)$ is slowly oscillating (in the sense of Stanojević), then

$$
\bar{d}\left(X_{m n}, L\right)<\epsilon, \quad m, n \rightarrow \infty .
$$

Proof. Let $X=\left(X_{m n}\right)$ be slowly oscillating, then $\sigma_{m n}^{(k, r)}(X)$ is slowly oscillating (by Lemma 1). Furthermore, since $X=\left(X_{m n}\right)$ is $(C, k, r)$-summable to a fuzzy number $L$, so by Theorem 3.1

$$
\bar{d}\left(\sigma_{m n}^{(k, r)}(X), L\right)<\epsilon, \quad m, n \rightarrow \infty .
$$

Next from the definition,

$$
\sigma_{m n}^{(k, r)}(X)=\sigma_{m n}^{(1,1)}(X)\left(\sigma_{m n}^{(k-1, r-1)}(X)\right) .
$$


Clearly, equation (3.8) and (3.9) implies $X=\left(X_{m n}\right)$ is $(C, k-1, r-1)$-summable to a fuzzy number $L$. Again $\left(\sigma_{m n}^{(k-1, r-1)}(X)\right)$ is also slowly oscillating (by Lemma 3.1); thus by Theorem 3.1, we have

$$
\bar{d}\left(\sigma_{m n}^{(k-1, r-1)}(X), L\right)<\epsilon, \quad m, n \rightarrow \infty .
$$

Continuing in this way, we obtain

$$
\bar{d}\left(X_{m n}, L\right)<\epsilon, \quad m, n \rightarrow \infty .
$$

Which completes the proof of the Corollary 3.1.

Remark 3.1. If $k=0$, and $r \neq 0$, then $(C, k, r)$ - summability reduces to $(C, 0, r)$ summability. Again for $k \neq 0$ and $r=0,(C, k, r)$-summability reduces to $(C, k, 0)$ summability.

Theorem 3.2. If the double sequence $X=\left(X_{m n}\right)$ of fuzzy number is $(C, 1,1)$ summable to a fuzzy number $L$ and $Y_{m n}^{(1,1)}\left(\Delta_{m n} u_{m n}\right)$ is slowly oscillating, then

$$
\bar{d}\left(X_{m n}, L\right)<\epsilon, \quad m, n \rightarrow \infty .
$$

Proof. As $\left(X_{m n}\right)$ is $(C, 1,1)$-summable to a fuzzy number $L$, so $\left(\sigma_{m n}^{(1,1)}\right)$ is $(C, 1,1)$ summable to a fuzzy number $L$. Therefore, $\left(Y_{m n}^{(1,1)}\right)$ is $(C, 1,1)$-summable to zero by equation (2.4). Using identity (2.4) to $\left(Y_{m n}^{(1,1)}\right)$, we get $Y\left(Y_{m n}^{(1,1)}\right)$ is Cesàro summable to zero. So that $Y\left(Y_{m n}^{(1,1)}\right)$ is oscillating slowly by Lemma 3.1. Now by Lemma 3.2(i),

$$
\begin{aligned}
& \bar{d}\left(Y\left(Y_{m n}^{(1,1)}\right), \sigma_{m n}^{(1,1)} Y\left(Y_{m n}^{(1,1)}\right)\right) \\
= & \frac{([\lambda m]+1)([\lambda n]+1)}{([\lambda m]-m)([\lambda n]-n)}\left[\bar{d}\left(\sigma_{[\lambda m],[\lambda n]}^{(1,1)} Y\left(Y_{m n}^{(1,1)}\right), \sigma_{[\lambda m], n}^{(1,1)} Y\left(Y_{m n}^{(1,1)}\right)\right)\right. \\
& \left.-\bar{d}\left(\sigma_{m,[\lambda n]}^{(1,1)} Y\left(Y_{m n}^{(1,1)}\right), \sigma_{m n}^{(1,1)} Y\left(Y_{m n}^{(1,1)}\right)\right)\right] \\
& +\frac{[\lambda m]+1}{[\lambda n]-m} \bar{d}\left(\sigma_{[\lambda m], n}^{(1,1)} Y\left(Y_{m n}^{(1,1)}\right), \sigma_{m, n}^{(1,1)} Y\left(Y_{m n}^{(1,1)}\right)\right) \\
& +\frac{[\lambda n]+1}{[\lambda m]-m} \bar{d}\left(\sigma_{m,[\lambda n]}^{(1,1)} Y\left(Y_{m n}^{(1,1)}\right), \sigma_{m n}^{(1,1)} Y\left(Y_{m n}^{(1,1)}\right)\right) \\
& -\frac{1}{([\lambda m]-m)([\lambda n]-n)} \bar{d}\left(\sum_{i=m+1}^{[\lambda m]} \sum_{j=n+1}^{[\lambda n]}\left(Y\left(Y_{i j}^{(1,1)}\right), Y\left(Y_{m n}^{(1,1)}\right)\right)\right) .
\end{aligned}
$$

It is easy to verify that for $\lambda>1$ and sufficiently large $n$

$$
\frac{([\lambda m]+1)([\lambda n]+1)}{([\lambda m]-m)([\lambda n]-n)}<\frac{([\lambda m]+1)([\lambda n]+1)}{([\lambda m]-1-m)([\lambda n]-1-n)}<\frac{4 \lambda^{2}}{(\lambda-1)^{2}} .
$$


Next, by (3.10)

$$
\begin{aligned}
\bar{d}\left(Y\left(Y_{m n}^{(1,1)}\right), \sigma_{m n}^{(1,1)} Y\left(Y_{m n}^{(1,1)}\right)\right) \leqq & \frac{4 \lambda^{2}}{(\lambda-1)^{2}} \bar{d}\left(\tau_{m n} Y\left(Y_{m n}^{(1,1)}\right), \sigma_{[\lambda m],[\lambda n]}^{(1,1)} Y\left(Y_{m n}^{(1,1)}\right)\right) \\
& -\max _{m+1, n+1 \leqq i, j \leqq[\lambda m],[\lambda n]} \bar{d}\left(\sum_{j=n+1}^{[\lambda n]}\left(Y\left(Y_{i j}^{(1,1)}\right), Y\left(Y_{m n}^{(1,1)}\right)\right)\right) .
\end{aligned}
$$

Taking limsup both sides of (3.11) we have

$$
\begin{aligned}
& \limsup _{m, n} \bar{d}\left(Y\left(Y_{m n}^{(1,1)}\right), \sigma_{m n}^{(1,1)} Y\left(Y_{m n}^{(1,1)}\right)\right) \\
\leqq & \frac{4 \lambda^{2}}{(\lambda-1)^{2}} \limsup _{m, n} \bar{d}\left(\tau_{m n} Y\left(Y_{m n}^{(1,1)}\right), \sigma_{[\lambda m],[\lambda n]}^{(1,1)} Y\left(Y_{m n}^{(1,1)}\right)\right) \\
& -\limsup _{m, n} \max _{m+1, n+1 \leqq i, j \leqq[\lambda m],[\lambda n]} \bar{d}\left(\sum_{j=n+1}^{[\lambda n]}\left(Y\left(Y_{i j}^{(1,1)}\right), Y\left(Y_{m n}^{(1,1)}\right)\right)\right) .
\end{aligned}
$$

Furthermore, as $\sigma_{[\lambda m],[\lambda n]}^{(1,1)} Y\left(Y_{m n}^{(1,1)}\right)$ converges, so first term in the right hand side of equation (3.12), must vanish.

This implies,

$$
\begin{aligned}
& \limsup _{m, n} \bar{d}\left(Y\left(Y_{m n}^{(1,1)}\right), \sigma_{m n}^{(1,1)} Y\left(Y_{m n}^{(1,1)}\right)\right) \\
\leqq & \limsup _{m, n} \max _{m+1, n+1 \leqq i, j \leqq[\lambda m],[\lambda n]} \bar{d}\left(\sum_{j=n+1}^{[\lambda n]}\left(Y\left(Y_{i j}^{(1,1)}\right), Y\left(Y_{m n}^{(1,1)}\right)\right)\right) .
\end{aligned}
$$

As $\lambda \rightarrow 1^{+}$in $(3.13)$, so we get

$$
\limsup _{m, n} \bar{d}\left(Y\left(Y_{m n}^{(1,1)}\right), \sigma_{m n}^{(1,1)} Y\left(Y_{m n}^{(1,1)}\right)\right) \leqq 0 .
$$

It implies that,

$$
\bar{d}\left(Y\left(Y_{m n}^{(1,1)}\right), 0\right)<\epsilon, \quad m, n \rightarrow \infty .
$$

Since $\left(X_{m n}\right)$ is summable to a fuzzy number $L$ by $(C, 1,1)$ mean and

$$
\bar{d}\left(Y\left(Y_{m n}^{(1,1)}\right), 0\right)<\epsilon, \quad m, n \rightarrow \infty
$$

so,

$$
\bar{d}\left(X_{m n}, L\right)<\epsilon, \quad m, n \rightarrow \infty .
$$

Which completes the proof of the Theorem 3.2.

Corollary 3.2. If $\left(X_{m n}\right)$ is $(C, k, r)$-summable to a fuzzy number $L$ and $Y_{m n}^{(1,1)}(\Delta X)$ is slowly oscillating, then

$$
\bar{d}\left(X_{m n}, L\right)<\epsilon, \quad m, n \rightarrow \infty .
$$


Proof. As $Y_{m n}^{(1,1)}(\Delta X)$ is slowly oscillating, setting $X=\left(X_{m n}\right)$ in place of $Y_{m n}^{(1,1)}(\Delta X)$, then $\sigma_{m n}^{(k, r)}\left(Y_{m n}^{(1,1)}(\Delta X)\right)$ is slowly oscillating by Lemma 3.1. Again as $Y_{m n}^{(1,1)}(\Delta X)$ is $(C, k, r)$-summable to a fuzzy number $L$, so by Theorem 3.2 , we have

$$
\bar{d}\left(\sigma_{m n}^{(k, r)}\left(Y_{m n}^{(1,1)}(\Delta X)\right), L\right)<\epsilon, \quad m, n \rightarrow \infty .
$$

By definition,

$$
\sigma_{m n}^{(k, r)}\left(Y_{m n}^{(1,1)}(\Delta X)\right)=\sigma_{m n}^{(1,1)}\left(Y_{m n}^{(1,1)}(\Delta X)\right)\left[\sigma_{m n}^{(k-1, r-1)}\left(Y_{m n}^{(1,1)}(\Delta X)\right)\right] .
$$

From (3.15) and (3.16) we have $Y_{m n}^{(1,1)}(\Delta X)$ is $(C, k-1, r-1)$-summable to a fuzzy number $L$. Again by Lemma 3.1, since

$$
\sigma_{m n}^{(k-1, r-1)}\left(Y_{m n}^{(1,1)}(\Delta X)\right)
$$

is slowly oscillating, so we have

$$
\bar{d}\left(\sigma_{m n}^{(k-1, r-1)}\left(Y_{m n}^{(1,1)}(\Delta X)\right), L\right)<\epsilon \quad \text { (by Theorem 3.2). }
$$

Continuing in this way, we obtain

$$
\bar{d}\left(\left(Y_{m n}^{(1,1)}(\Delta X)\right), L\right)<\epsilon, \quad m, n \rightarrow \infty .
$$

Which completes the proof of the Corollary 3.2 .

Remark 3.2. If $k=0$, and $r \neq 0$, then $(C, k, r)$ - summability reduces to $(C, 0, r)$ summability. Again for $k \neq 0$ and $r=0,(C, k, r)$-summability reduces to $(C, k, 0)$ summability and consequently the following corollaries are generated from the main result.

\section{REFERENCES}

[1] Y. Altın, M. Mursaleen and H. Altinok, Statistical summability $(c, 1)$ for sequences of fuzzy real numbers and a tauberian theorem, Journal of Intelligent and Fuzzy Systems 21 (2010), 379-384.

[2] I. Çanak, Tauberian theorems for cesàro summability of sequences of fuzzy numbers, Journal of Intelligent and Fuzzy Systems 27 (2014), 937-942.

[3] H. Dutta, On some complete metric spaces of strongly summable sequences of fuzzy numbers, Rend. Semin. Mat. Univ. Politec. Torino 68 (2010), 29-36.

[4] H. Dutta, A characterization of the class of statistically pre-Cauchy double sequences of fuzzy numbers, Appl. Math. Inf. Sci. 7 (2013), 1433-1436.

[5] H. Dutta and F. Başar, A generalization of orlicz sequence spaces by cesàro mean of order one, Acta Math. Univ. Comenian. (N.S.) 80 (2011), 185-200.

[6] H. Dutta and T. Bilgin, Strongly $\left(v^{\lambda}, a, \delta_{v m}^{n}, p\right)$-summable sequence spaces defined by an orlicz function, Appl. Math. Lett. 24 (2011), 1057-1062.

[7] B. B. Jena, S. K. Paikray and U. K. Misra, A tauberian theorem for double cesàro summability method, Int. J. Math. Math. Sci. 2016 (2016), 1-4.

[8] B. B. Jena, S. K. Paikray and U. K. Misra, Inclusion theorems on general convergence and statistical convergence of $(l, 1,1)$-summability using generalized tauberian conditions, Tamsui Oxf. J. Inf. Math. Sci. 31 (2017), 101-115.

[9] K. Knopp, Limitierungs-umkehrsätze für doppelfolgen, Math. Z. 45 (1939), 573-589.

[10] M. Matloka, Sequences of fuzzy numbers, Busefal 28 (1986), 28-37.

[11] F. Móricz, Tauberian theroems for cesàro summable double sequences, Studia Math. 110 (1994), $83-96$. 
[12] S. Nanda, On sequences of fuzzy numbers, Fuzzy Sets and Systems 33 (1989), 123-126.

[13] P. V. Subrahmanyam, Cesàro summability of fuzzy real numbers, J. Anal. 7 (1999), 159-168.

[14] O. Talo and F. Başar, On the slowly decreasing sequences of fuzzy numbers, Abstr. Appl. Anal. 2013 (2013), 1-7, Article ID 891986.

[15] O. Talo and C. Çakan, On the cesàro convergence of sequences of fuzzy numbers, Appl. Math. Lett. 25 (2012), 676-681.

[16] B. C. Tripathy and S. Debnath, On generalized difference sequence spaces of fuzzy numbers, Acta Scientiarum Technology 35 (2013), 117-121.

[17] B. C. Tripathy, A. Baruah, M. Et and M. Gungor, On almost statistical convergence of new type of generalized difference sequence of fuzzy numbers, Iran. J. Sci. Technol. Trans. A Sci. 36 (2012), 147-155.

[18] Č. V. Stanojević, Analysis of divergence control and management of divergent process, in: $\dot{\mathrm{I}}$. Çanak (Ed.), Graduate Research Seminar Lecture Notes, University of Missouri-Rolla, Fall, 1998.

[19] L. A. Zadeh, Fuzzy sets, Information and Control 8 (1965), 338-353.

${ }^{1}$ Department of Mathematics,

Veer Surendra Sai University of Technology,

Burla 768018, Odisha, India

Email address: bidumath.05@gmail.com

Email address: skpaikray_math@vssut.ac.in

${ }^{2}$ Research Scholar Department of Mathematics,

RAVENSHAW UNIVERSITY,

CutTack 753003, Odisha, India

Email address: priyadarsiniparida1@gmail.com

${ }^{3}$ Department of Mathematics,

Gauhati University,

GuWAhati 781014, Assam, InDia

Email address: hemen_dutta080rediffmail.com 